\title{
The Charge Carrier Density Influence and Thermal Effects on Charge Transport in Organic Semiconductors
}

\author{
Sara Santiago de Brito, Wiliam Ferreira da Cunha, Demétrio Antônio da Silva Filho \& \\ Pedro Henrique de Oliveira Neto
}

\section{Introduction}

Conjugated polymers have optical and electronic properties presenting semiconductor characteristics that make them ideal for manufacture of solar cells and electronic devices. These organic materials may be similar to the metals and semiconductors. Peculiar qualities are related to processes involved in interchain charge transport. In this sense, a phenomenological description involving temperature, different types of charge carriers, as well as, the influence of charge carriers density is fundamental to the understanding and eventual improvement of these devices. Usually, the creation of charge carriers in organic conductors is connected to photon absorption or injection of electrons in gaps, due to the large electron-phonon interaction, which leads to creation of quasiparticles. These particles are responsible for the charge transport in conjugated polymers. Recently, in a theoretical study, it was observed the temperature effect on interchain polarons dynamics. The results suggest a charge transfer increase due to the thermal effects leading to greater mobility of the carriers ${ }^{1}$. In another study, the effect of charge carriers density was studied, showing that charge density increase leads to a nonlinear carrier mobility growth ${ }^{2}$. However, both studies take into account only polarons. Therefore, the thermal effect on interchain charge transport remains poorly described.

In this research, we study the charge transport between two polyacetylene molecules in the presence of external electric field. We take into account several temperature regimes, electric field and charge density. We have investigated the both polarons and bipolarons quasiparticles and the influence of intermolecular interaction for transportation of charge in coupled chains. This study deals with the improvement of interchain charge transport.

\section{Methods}

We use a modified version of model Su-SchriefferHeeger ( $\mathrm{SSH}$ ). The model is a semi-empirical tightbinding which considers the interactions between the nearest neighboring sites where the network potential are weakly overlapping. The dynamic of the system was solved on a numerical approach in the context of Hartree-Fock approximation. The SSH model was modified to include the effects of temperature, the term of Brazovskii-Kirova symmetry breaking, external electric field and interaction between chains. We use the following Hamiltonian:

$$
H=H_{1}+H_{2}+H_{\text {int }}
$$

with $j$ indexing the chain, and:

$$
\begin{gathered}
H_{j}=\sum_{n, s} t_{j n, n+1}\left(C_{j n+1, s}^{\dagger} C_{j n, s}+\text { H.c. }\right)+ \\
+\sum_{n} \frac{\mathrm{K}}{2} \mathcal{Y}_{j n}^{2}+\sum_{n} \frac{p_{j n}^{2}}{2 M^{\prime}}
\end{gathered}
$$


The operator $\mathrm{C}_{\mathrm{jn}, \mathrm{s}}$ is the annihilation operator of an $\pi$ electron with spin $s$ at the $n$-th site of the $j$-th chain, $\mathrm{K}$ is the harmonic constant and $\mathrm{M}$ is the mass of a $\mathrm{CH}$ group. The Hopping term is $\mathrm{t}_{\mathrm{jn}, \mathrm{n}+1}=\left[1+(-1) n \delta_{0}\right]\left(\mathrm{t}_{0}+\mathrm{y}_{\mathrm{jn}}\right)$, where $\alpha$ is the electron-phonon coupling constant, $t_{0}$ is the transfer integral between the nearest neighboring sites in an undimerized chain and $\delta_{0}$ is the BrazovskiiKirova symmetry breaking parameter.

\section{Results and Discussion}

The simulations in this study describe two coupled cis-polyacetylene chains with 80 sites each ${ }^{3}$.
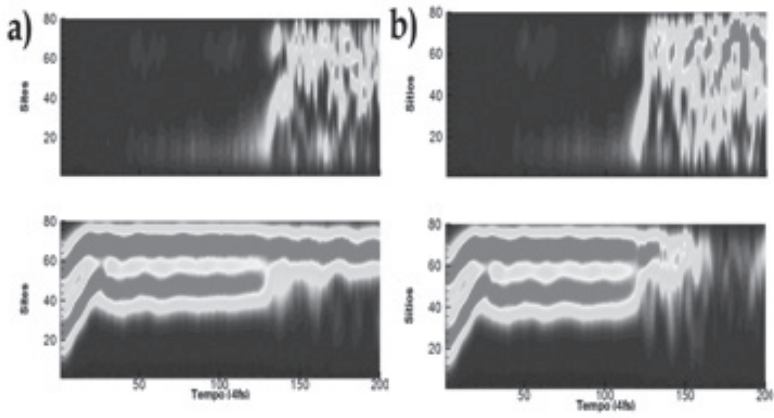

Figure 1: Temporal evolution of charge density for two polarons for an electric field of $-0,0195 \mathrm{mV} / \AA$ : (a) $\mathrm{T}=0 \mathrm{~K}$, (b) $\mathrm{T}=4,7 \mathrm{~K}$.
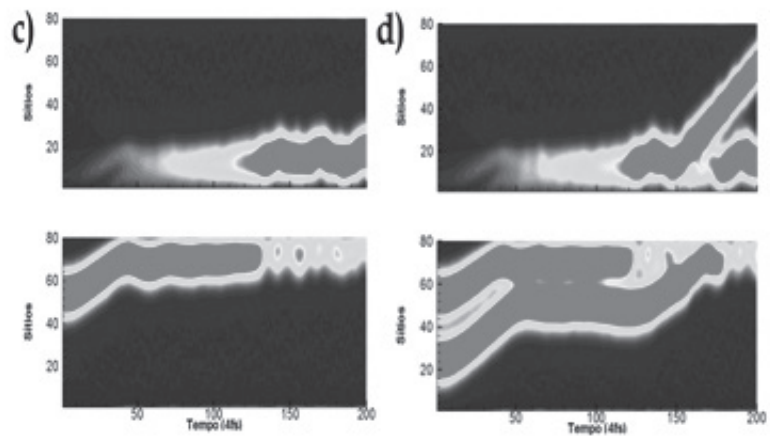

Figure 2: Temporal evolution of charge density for two bipolarons for an electric field of $-0,013 \mathrm{mV} / \AA$ : (c) $\mathrm{T}=50 \mathrm{~K}$, (d) $\mathrm{T}=100 \mathrm{~K}$.
The temperature regime ranged from 0 to $100 \mathrm{~K}$. The results are shown respectively in the graphs of Figures 1(a), 1(b) and 2(c), 2(d). In the first set of simulations figure 1.a) shows two polarons in the presence of an external electric field at $\mathrm{T}=0 \mathrm{~K}$. Eventually, these quasiparticles reach the end of the chain where there is the interaction between the molecules. Due to the strong electrical field, one of the quasiparticles moves to the next chain. However, the charge density profile suggests the degradation of the polaron. Here, the external electric field provides energy in order to dissociate the charge from the phonons. Figure 1.b) shows the 4,7 K temperature regime. It is noted that, in the presence of thermal effects, the charge transfer occurs faster than in the absence of temperature, however both quasiparticles have degraded. The figure 2 shows the interchain charge transport with bipolarons. 2-c) shows one bipolaron in the interface when thermal effects are taken into account. With this charge carrier, the energy provided by the electric field and the temperature were not enough to overcome the barrier between the two molecules. In 2-d) it is presented a simulation with two bipolarons. Note that the increase in charge density initiates the interchain charge carrier transport mechanism. Also, we find that an increase in the temperature do not change this mechanism.

\section{Conclusions}

It was investigated the interchain charge transport in polyacetylene molecules. In order to do this, it was used o modified version of SSH model. Both temperature and carriers density play an important role on charge transport in conjugated polymers.

In this study we analyzed different charge carriers. We simulated conjugated-polymer chains with polarons and bipolarons under the presence of an external electric field and thermal effects. The simulations investigated the interchain charge transport. The results of simulations performed in this study reveal for two polarons the effect of interaction between quasiparticles affects the charge transport. The interaction between two bipolarons results in charge transportation between chains and effective untrapping area of interaction interchain. It is possible to visualize how the thermal effects provide charge carrier mobility gain. We observed that the perturbation provided by thermal effects improves the charge delocalization. 
Besides, in a interchain process the results suggest that the polaron transfer is enhanced by thermal perturbation.

In this study, it is presented the temperature increase raises the delocalization of the charge carrier, fastening the transport and, thus, leading to a mobility increase. It was shown that temperature effects give rise to systems with an improved of charge carriers and higher mobility especially through untrapping mechanisms.

\section{Acknowledgments}

The authors thank the funding agencies CNPq, CAPES and Finatec.

\section{References}

1. P. H. D. Neto, W. F. Cunha and G. M. e Silva. Europhys. Lett. 88, 67006 (2009).

2. P. H. D. Neto, W. F. Cunha, R. Gargano and et. al.. J. Phys. Chem. A. 113, 14975 (2009).

3. S.S. Brito, Influência da Densidade de Portadores e de Efeitos Térmicos no Transporte de Carga em Semicondutores Orgânicos. Dissertação de mestrado, Brasília, 2014.

\section{Sara S. de Brito*, Wiliam F. da Cunha, Demétrio A. da Silva Filho \& Pedro H. de Oliveira Neto}

Instituto de Física, Universidade de Brasília. CP04455, Brasília, DF, CEP 70919-970, Brasil.

*E-mail: sarahsantiagobrito@gmail.com 